\title{
Experiencias
}

\section{Transitando por la pandemia: adaptaciones a la epidemia de la covid-19 desde el programa de Atención Temprana del CRE de Sevilla}

Implications of the pandemic: adaptations to the covid-19 epidemic at the Seville Educational Resource Centre's early care programme

\section{García-Trevijano Patrón, M. C. Hernández Mariano, C. P. Regidor Poyatos, J. Gómez Mateos, M. M. Martín de Frutos}

\section{Resumen}

En este documento se expone el recorrido del programa de Atención Temprana (AT) del Centro de Recursos Educativos (CRE) de la ONCE en Sevilla desde el inicio del confinamiento a la actualidad. La adaptación a las dificultades iniciales al iniciar el teletrabajo, la elaboración de nuevos protocolos y el mantenimiento de algunas actividades de forma telemática con las familias han permitido seguir desarrollando nuestra intervención en esta realidad cambiante provocada por la covid-19. Esta situación ha supuesto un enorme esfuerzo, físico y también emocional, tanto para los profesionales como para las familias, tratando de conseguir como objetivo último el mantenimiento de la calidad en la atención que prestamos a estas, junto a sus bebés y niños pequeños.

\section{Palabras clave}

Atención Temprana. Discapacidad visual. Estado emocional. Confinamiento. Trabajo en equipo. Familias. Adaptación de material educativo. Protocolos de actuación. 


\begin{abstract}
This article describes the changes adopted by the early care unit working out of ONCE'S Educational Resource Centre at Seville between institution of lockdown and this writing. By adapting to the initial difficulties posed by remote working, establishing new protocols and conducting activities to engage with families in IT format, the unit was able to gear its operations to the changing realities prompted by covid-19. The new situation called for an enormous physical and emotional effort on the part of professionals and families alike to maintain the quality of care provided parents, their babies and young children.
\end{abstract}

\title{
Key words
}

Early care. Visual disability. Emotional status. Lockdown. Teamwork. Families. Adaptation of educational material. Action protocols.

\section{Introducción}

Recogemos en este documento el recorrido del programa de Atención Temprana ${ }^{1}$ del CRE de Sevilla desde el inicio del confinamiento a la actualidad. Muchos son los aspectos que definirían la adaptación a la pandemia, unos más técnicos y otros más personales, en un tiempo necesariamente teñido de emociones. Hemos querido combinar ambos, con la convicción de que, solo desde la conciencia y el análisis de la situación que estamos viviendo, tendrá sentido el enorme esfuerzo de tantos profesionales y familias. Todo ello, con un objetivo común: mantener entornos afectuosos y estimulantes para los niños y niñas con discapacidad visual en sus primeras edades, entornos que les permitan seguir creciendo de forma segura. Y con la confianza de dejar pronto atrás lo que en boca de nuestros bebés sería «Aquel tiempo en el que no podíamos soplar pompas». ${ }^{2}$

Pensamos que nuestro trabajo no es sino el inicio de una cadena donde muchas personas estamos implicadas, empezando por las familias de los bebés con discapacidad

\footnotetext{
1 Se define la Atención Temprana (AT) como la intervención dirigida a niños de 0 a 6 años -y sus familias - que padecen o tienen riesgo de padecer alguna discapacidad. Se concreta en actuaciones dirigidas al niño, a su familia y al entorno. 2 Hemos querido poner este ejemplo porque pensamos que soplar pompas, seguirlas en su movimiento y tocar su fragilidad, es una de las actividades más placenteras para todos los bebés, tengan o no cualquier discapacidad. Esto incide en dos aspectos que hemos tenido que dejar de hacer en la adaptación a esta pandemia: no podemos quitarnos la mascarilla, no podemos soplar y, por lo tanto, no podemos ayudarles a construir pompas. Nos perdemos la magia de su asombro al verlas flotar o cuando les hacen cosquillas en las manos y en el rostro.
} 
visual y creciendo a su alrededor multitud de profesionales de todos los ámbitos, desde dentro y fuera de una institución como la ONCE, que trabaja, cada vez más, trabaja como miembro activo de un mundo en cambio continuo, cambio sostenido también por todos y cada uno de sus trabajadores.

Es por esto que queremos ser testigos de este tiempo, mostrando cómo hemos vivido esta experiencia, sorteando el exceso de trabajo, el cansancio, la tristeza y, por qué no, el amor a la tarea y a la pertenencia a un equipo. Por ello, a veces, en la redacción de este artículo mantenemos la primera persona y las impresiones personales. Describimos dicha experiencia en dos periodos: el primero, durante el confinamiento, que extenderíamos hasta el final de curso 2019-2020, tiempo marcado por el trabajo virtual; el segundo, desde el inicio de este curso 2020-2021 hasta la actualidad, justo al año del comienzo de la epidemia de covid-19, tiempo marcado por la convivencia del trabajo presencial con el virtual.

\section{Primer periodo}

Al inicio del confinamiento, ante una situación nueva e imprevista, caracterizada por la brusquedad del cambio de una situación presencial a otra virtual, nos planteamos cómo seguir trabajando. Ante esta pregunta, surge, de manera casi inmediata, la idea de anclar el trabajo virtual al que ya se estaba realizando. Se decide así mantener la organización básica del trabajo presencial, realizando las adaptaciones que la situación y el modo de intervención han requerido.

En definitiva, se ha mantenido la estructura original del trabajo en equipo, aunque de manera virtual, a través de:

- La continuidad de las reuniones semanales de coordinación de AT.

- La organización de las sesiones, respetando el horario presencial habitual y flexibilizándolo en función de las necesidades de cada familia.

- El empeño en el cumplimiento de los criterios de calidad que nos planteamos para el programa.

Se pueden concretar dichas actuaciones en cuatro ámbitos: 
- Con la familia:

Se ha trabajado online. La mayoría de las familias se han incorporado con mucha eficacia a la atención virtual, y algunas han mostrado menor necesidad de apoyo. Los vídeos que estas nos remitían, mostrando actividades y situaciones cotidianas con sus hijos, nos han permitido abordar las cuestiones de desarrollo y de crianza que les preocupaban. No todas han mostrado las mismas necesidades, aspecto que se ha respetado, así como las situaciones inesperadas que han ido surgiendo.

Tenemos que destacar que se han realizado, de forma telemática, la acogida a las familias y la valoración de los bebés que se han incorporado al programa en este periodo. Este aspecto ha sido muy complejo $y$, aunque no ha podido completarse por razones obvias, ha permitido el acompañamiento en estos momentos iniciales tan complicados. Somos conscientes ahora de los lazos tan estrechos que se han creado con estas familias.

- Con los niños y niñas:

La edad de la población atendida ha determinado las adaptaciones y la necesidad de la participación activa de las familias. En este sentido, pensamos que el paso al teletrabajo se ha facilitado gracias a que nuestro modelo de intervención se estructura desde la interacción familiar, por lo que estas han podido adaptarse con facilidad a las propuestas planteadas. ${ }^{3}$ Con los más pequeños hemos necesitado la mediación de los padres y madres. Mientras que con algunos de los alumnos y alumnas del segundo ciclo de Educación Infantil (de 3 a 6 años) se ha podido trabajar como interlocutores activos.

Resaltamos lo complejo del abordaje en aquellas situaciones en las que, junto a la discapacidad visual severa, existe un problema grave de comunicación. Esto ha obligado a crear nuevas propuestas, donde las familias puedan conectar con las dinámicas ya iniciadas en la atención presencial. En concreto, se ha desarrollado un programa de proyectos semanales, basados en la propia experiencia del niño, que han requerido la participación activa de los padres y la anticipación (Figura 1). Este nuevo enfoque ha sido valorado positivamente por los padres de los alumnos.

3 En nuestro programa de AT partimos de un modelo de intervención con el niño a través de su familia y en el entorno natural de crianza, a diferencia de otros modelos que inciden más en la intervención directa sobre el bebé. Así pues, ha sido fácil trasladar a la casa lo que ya hacíamos presencialmente. 
Figura 1. Captura de pantalla de una sesión online

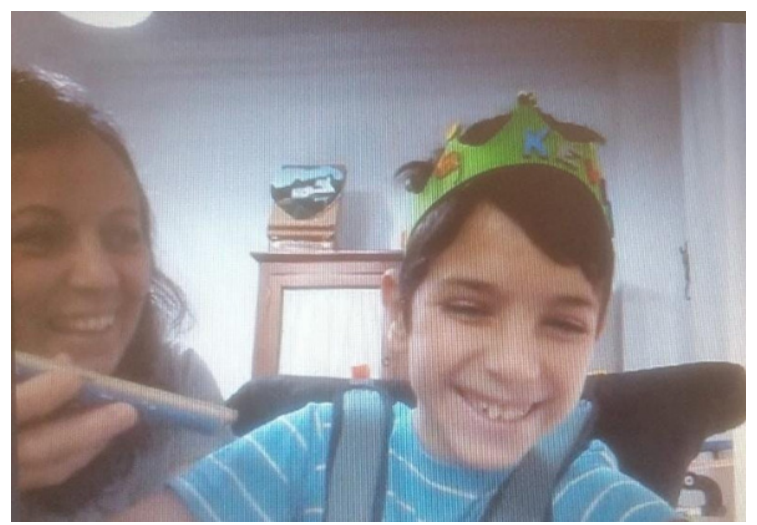

- En los casos de niños con sordoceguera, ${ }^{4}$ se ha trasladado la metodología específica a las familias, integrando la anticipación con objetos de referencia a su rutina diaria en casa. Se han acordado con ellos los objetos y los gestos/signos adecuados para la comunicación, enviando vídeos explicativos de los signos y canciones o cuentos signados (Figura 2).

Figura 2. Captura de pantalla en sesión online trabajando Lengua de Signos Española

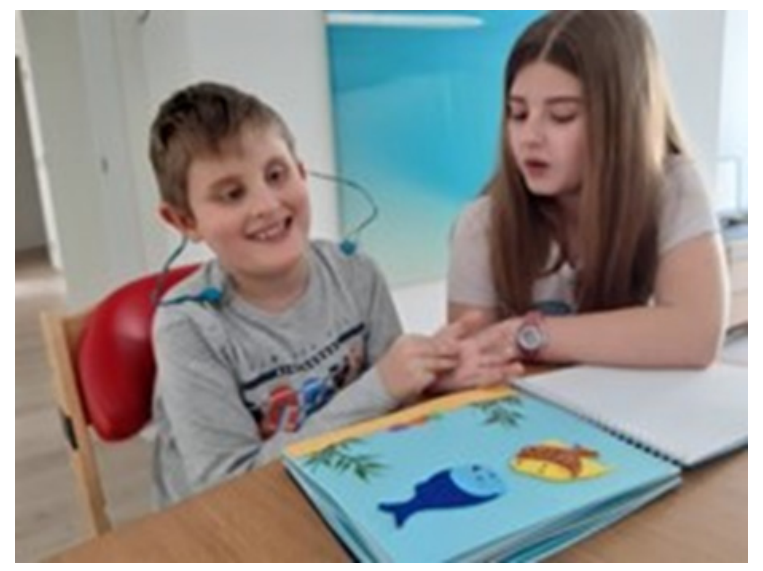

Ambos casos de problemas graves de comunicación, unidos a la ceguera o la condición de sordoceguera, han supuesto todo un reto a la hora de llevar a cabo nuestra intervención de manera telemática. El cariño, la confianza en el niño y el conocimiento de este, junto al apoyo incondicional de las familias, han permitido realizar

\footnotetext{
4 En nuestro programa atendemos a una población importante de alumnos con sordoceguera, es decir, pequeños que tienen déficit visual grave más discapacidad auditiva significativa. 
nuestro trabajo con eficacia, salvando las barreras de la distancia y la imposibilidad del contacto físico, fundamental en la relación con estos niños y niñas.

Este nuevo enfoque ha sido valorado positivamente tanto por las familias como por los profesionales del equipo.

- Con los profesionales:

Coincidimos en que, en esta situación de confinamiento, la colaboración y la coordinación han podido realizarse con mucha más eficacia. Y no solo entre los diferentes profesionales del equipo y del CRE, sino con todos los del ámbito de la atención a la primera infancia, tanto hospitalarios como de centros de AT y centros escolares. Esta situación es novedosa; recordamos que, habitualmente, en nuestras memorias hemos recogido dificultades de coordinación, aspecto que consideramos prioritario en el programa. Sin embargo, al ser presenciales, requerían una dedicación horaria de la que a veces no se disponía.

- Elaboración de materiales:

Figura 3. Violín, ${ }^{5}$ cuento adaptado de la editorial Kalandraka

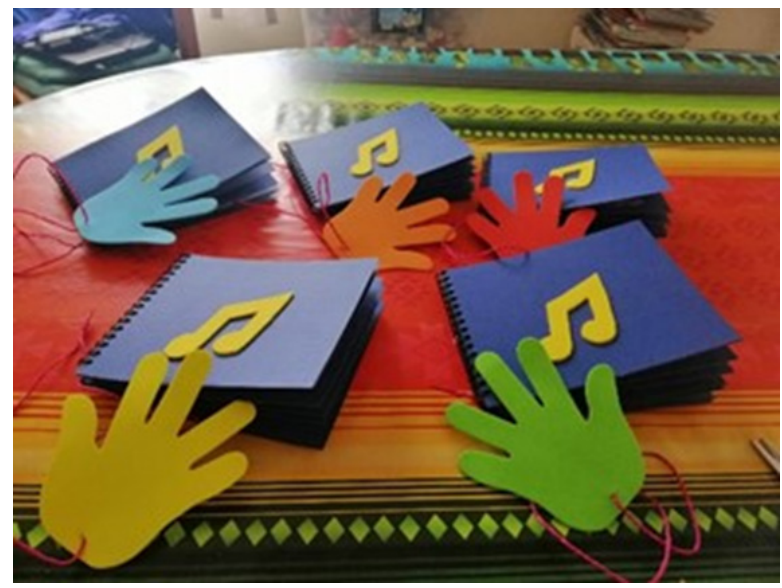

Hemos elaborado gran cantidad de materiales y recursos. Asimismo, se ha trabajado en la adaptación de juegos, cuentos y fichas, en ocasiones en coordinación con colegios y escuelas infantiles (Figuras 3 y 4). También se han

5 Escrito por Antonio Rubio e ilustrado por Óscar Villán, publicado por Kalandraka en 2018 (N. del E.). 
grabado canciones y cuentos en audio y vídeo, algunos de ellos en Lengua de Signos Española (LSE). Finalmente se ha puesto énfasis, dada la situación de confinamiento, en la elaboración de materiales artesanales con los recursos habitualmente disponibles en los domicilios.

Figura 4. Luna, ${ }^{6}$ cuento de la editorial Kalandraka

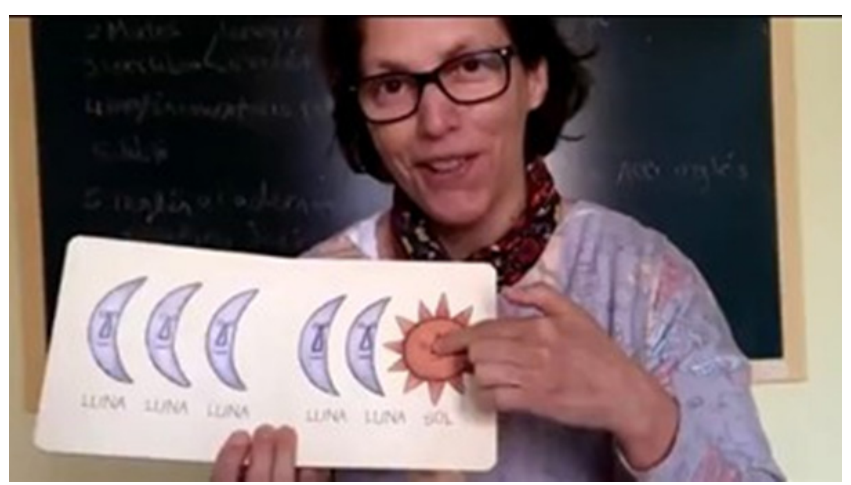

\subsection{Valoración de este primer periodo}

Mirando hacia atrás, valorando los meses de confinamiento, hay dos aspectos que nos hacen sentir más satisfechas. Por un lado, haber mantenido las actividades del programa y, por otro, el diseño y la elaboración de múltiples materiales, según las necesidades que se planteaban. Hemos podido adaptarnos a la situación de teletrabajo y a los elementos que nos han permitido desarrollarlo. Parecía imposible, pero entre todos se consiguió hacerlo.

Analizamos esta etapa de forma más concreta:

- La situación de confinamiento ha mostrado de forma más explícita la capacidad de los padres para incorporar en la crianza de sus hijos las pautas de actuación que trabajamos en las sesiones presenciales. Observamos que las familias que mostraban más implicación antes de esta situación, nos han hecho más demandas, y nuestro intercambio de información ha sido más fluido. Por otro lado, las que se implicaban menos han permanecido más pasivas, lo que ha hecho difícil la intervención. Aspecto que hemos considerado importante respetar.

6 Escrito por Antonio Rubio e ilustrado por Óscar Villán, publicado por Kalandraka en 2018 (N. del E.). 
- Valoramos muy positivamente haber podido mantener la atención de forma continuada, lo que ha significado para la mayoría de las familias un extraordinario soporte emocional. Este periodo ha supuesto un cambio drástico en su día a día, que ha acentuado su situación de vulnerabilidad. Esto adquiere especial importancia en situaciones de multidiscapacidad, donde los padres valoran con frecuencia que las diferentes terapias a las que acuden sus hijos les ayudan a sentirse sostenidos emocionalmente.

- Recogemos también la dificultad, expresada por las familias, para conciliar el teletrabajo con la atención a sus hijos, agravada cuando ambos progenitores han estado en esta situación.

- Finalizamos el curso realizando con las familias las actividades colectivas, actividades que favorecen el sentimiento de pertenencia al grupo. Destacamos este aspecto porque lo consideramos especialmente vulnerable dada la situación de confinamiento.

$Y$ en cuanto a los propios profesionales, coincidimos en la dificultad para concentrarnos, cerrar horarios y, por lo tanto, en la conciliación de la vida laboral y familiar. Esto se une, como no podía ser de otra manera, a la preocupación por la situación sanitaria global y los cambios que esta ha impuesto en lo cotidiano.

Hemos valorado la necesidad del trabajo social, desde la acogida de familias hasta toda la gestión de ayudas, en un momento tan complejo económica y socialmente. Pensamos, asimismo, que en el confinamiento se ha enfatizado la importancia del trabajo psicológico con aquellas, en estos momentos de cambio y gran ansiedad social. Recordamos la importancia de ambas figuras, cara a las necesidades que las familias nos han planteado día a día.

\section{Segundo periodo}

Este segundo periodo se inicia con el curso escolar, momento marcado claramente por la incertidumbre.

En el diseño general del Plan de Atención Temprana se propuso asumir la situación de pandemia. Para ello, se diseñaron actividades flexibles según los posibles escena- 
rios, siguiendo las directrices de la Dirección General de Educación, Empleo y Braille y de la coordinación del equipo.

Nuestra primera preocupación fue afianzar la atención presencial. Para ello, elaboramos un protocolo exhaustivo donde se recogen las diferentes normas a cumplir con el fin de mantener la mayor seguridad posible, tanto para los niños atendidos como para las familias y los profesionales.

En este protocolo se recogen aspectos relacionados con el proceso de atención durante las sesiones, incorporando las normas de protección anticovid y todos los aspectos de higiene en ellas implicados (Figuras 5,6 y 7 ).

Figura 5. Punto de higiene en sala de Atención Temprana

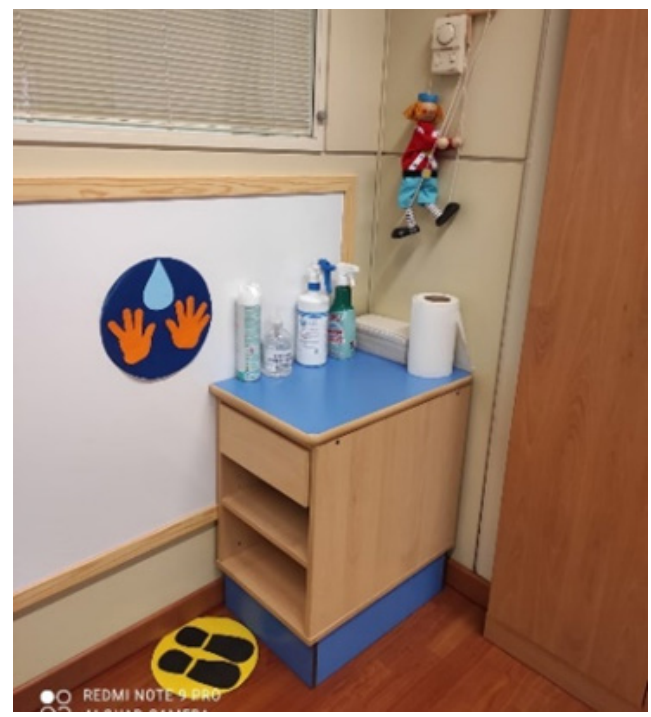

Figura 6. Señalización en puertas para indicar sala limpia-desinfectada o sala ya usada y que necesita limpieza y desinfección
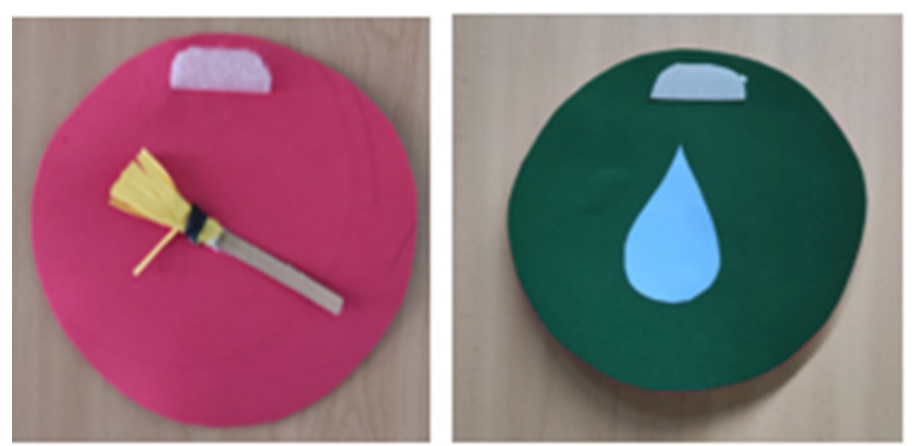

García-Trevijano, C., Hernández, M.C., Regidor, C.P., Gómez, J., Martín, M.M. (2021). Transitando por la pandemia: adaptaciones a la epidemia de la covid-19 desde el programa de Atención Temprana del CRE de Sevilla. RED Visual: Revista Especializada en Discapacidad Visual, 77, 182-199. https://doi.org/10.53094/TRIJ4448. 
Figura 7. Nos lavamos las manos antes de empezar

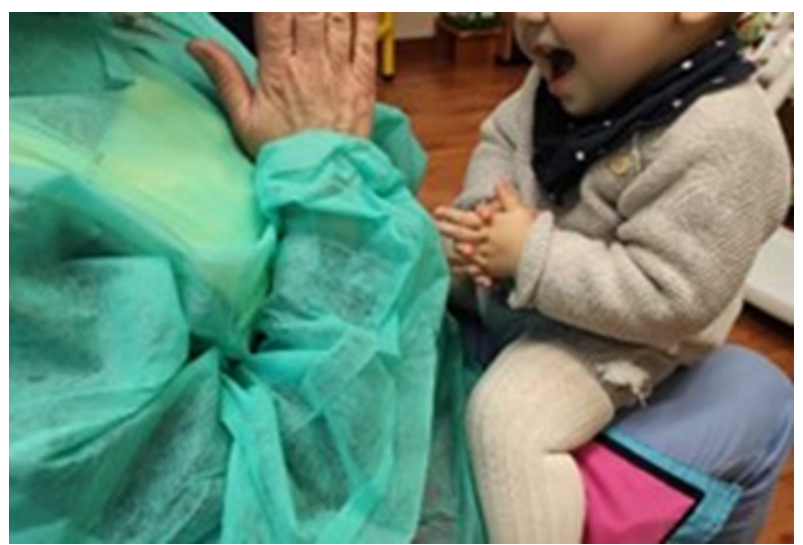

Además, se elaboró un documento donde se recuerdan los aspectos de protección y normas a cumplir, así como los recorridos de entrada y salida al CRE. Este documento se remitió a cada familia y se encuentra recogido en el plan del equipo.7

Se decidió imprescindible mantener a uno de los padres en las sesiones. Este criterio, que se analizó con especial detenimiento, es consecuencia lógica de un soporte teórico basado en los aspectos vinculares, según el cual consideramos a la familia protagonista principal de la intervención (Figura 8).

Figura 8. Sesión de Atención Temprana con la presencia de la familia

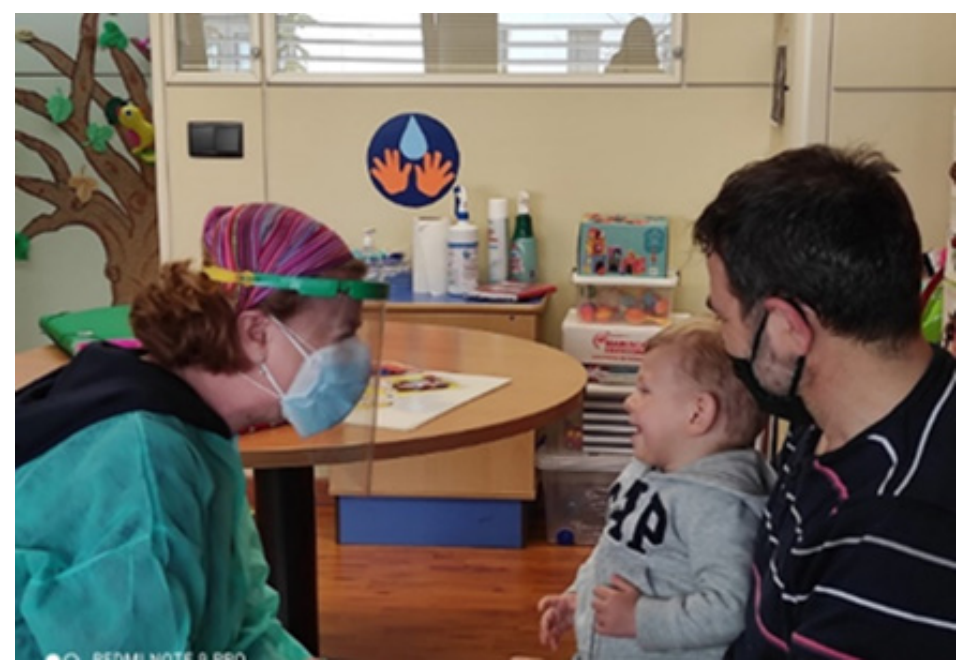

\footnotetext{
7 Para acceder a estos documentos de uso interno reflejados en el plan del equipo, sería necesario ponerse en contacto con el coordinador del Equipo Específico de Atención Educativa a alumnos con discapacidad visual.

García-Trevijano, C., Hernández, M.C., Regidor, C.P., Gómez, J., Martín, M. M. (2021). Transitando por la pandemia: adaptaciones a la epidemia de la covid-19 desde el programa de Atención Temprana del CRE de Sevilla. RED Visual: Revista Especializada en Discapacidad Visual, 77, 182-199. https://doi.org/10.53094/TRIJ4448.
} 
Destacamos también que, aunque estamos realizando nuestro trabajo en modalidad presencial, hay familias que nos han pedido hacerlo online o combinar ambos (Figura 9).

Figura 9. Sesión de Atención Temprana online

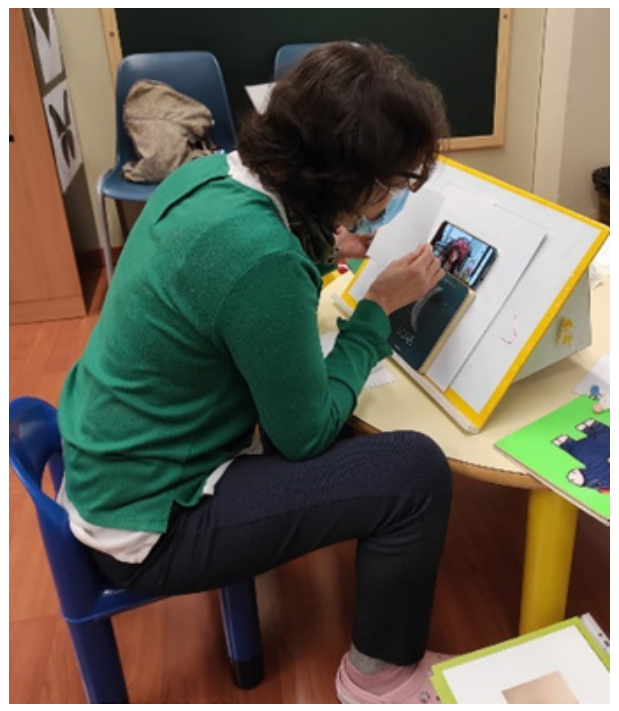

Esta adaptación del plan de Atención Temprana a la situación de pandemia enfatiza tres aspectos organizativos: la ventilación, la limpieza y desinfección de las salas y la coordinación de los diferentes horarios entre las profesionales de AT. Con este último, se pretende evitar la coincidencia de varias familias en la recepción y sus recorridos por el CRE.

La coordinación con recepción se hace aún más necesaria, sobre todo cuando es difícil ser estrictos con los horarios («¿Pueden subir?», nos preguntan desde recepción, o «Ahora sube, que está tomando su biberón»).

A veces, los bebés se duermen, o los papás y mamás necesitan desahogarse y esperamos un rato. Con ello, la persona que limpia se convierte en alguien más del equipo («¿Espero a que salgáis?», «Espera un poco, que ya acabamos»; ver Figura 10).

Recogemos, asimismo, que, en el contexto de adaptación a la pandemia y dada la importancia del uso de los espacios al aire libre, se propuso trasladar la sala de psicomotricidad a los espacios exteriores del CRE. Se tuvo que inhabilitar tras realizar el protocolo covid-19. Diseñamos lo que hemos llamado el ParCRE (ver Apéndice), que tiene como objetivo disponer de un lugar de juego al aire libre. Con él, se pretende 
mantener un espacio donde niños y niñas puedan disfrutar del movimiento, así como poder abordar aspectos de motricidad gruesa y de movilidad en exteriores.

Figura 10. Limpieza y desinfección de sala entre sesión y sesión

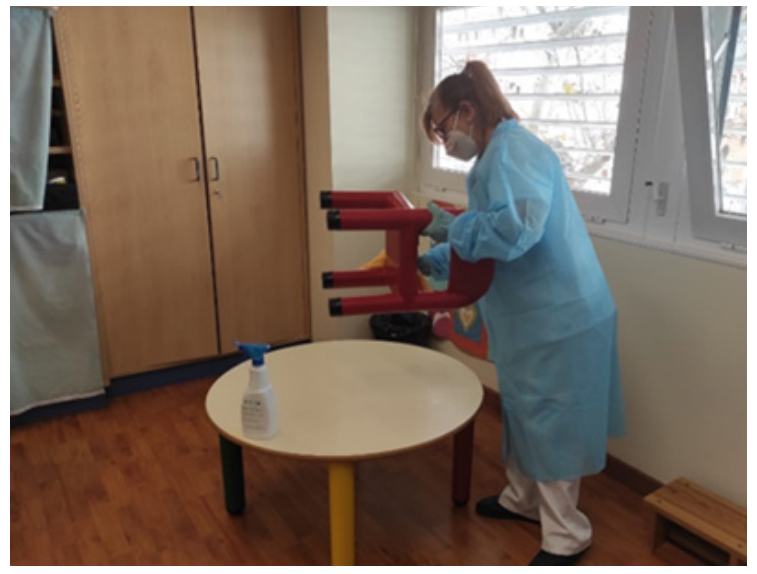

Otro de los objetivos claramente planteados desde la coordinación del programa fue mantener las actividades de grupo, entre ellas la actividad «Pequeartistas» (con la colaboración de la especialista de Plástica) y la «Merienda». ${ }^{8}$

De las actividades grupales, solo se ha suprimido una, la excursión con los niños de segundo ciclo de educación infantil. Esta actividad tiene como objetivo básico la convivencia entre niños y niñas de este ciclo, aspecto que la convierte en un ejemplo claro de lo que no puede adaptarse en esta situación de pandemia.

\subsection{Experiencia con el uso del formato digital}

Pasado un tiempo, podemos tener las ideas un poco más claras y acercarnos al uso de las herramientas digitales viendo sus pros y contras. Nuestro análisis de dicho formato, en cuanto a las actividades que realizamos, lo concretamos así:

\footnotetext{
8 La actividad de «Pequeartistas» consiste en que, con motivo de la Navidad, realizamos un encuentro de convivencia entre las familias con niños de 0-6 años donde realizamos alguna manualidad alusiva a estas fechas, dando a conocer a los padres la importancia de la manipulación y de la estimulación del tacto para sus hijos a través de la realización de alguna decoración navideña. Se orienta a los padres sobre la necesidad de compartir tiempo de calidad con los niños disfrutando de la actividad.

Las «meriendas» son dos encuentros realizados al principio y al final del curso con familias de niños de 0-6 años, con el objetivo de proporcionar un espacio de convivencia entre las familias. Los padres manifiestan la importancia de estos momentos que pueden compartir con otros con las mismas necesidades en un espacio de acogimiento y diversión.
} 
- En cuanto a las sesiones online, con los niños y sus padres:

Suelen ser por videollamadas de WhatsApp. Para su organización, concretamos una hora y un tiempo determinado de duración. Llegado el momento, avisamos de la llamada. Este tipo de sesiones requieren una cuidadosa preparación. Nos permiten interaccionar con el bebé, observar sus sonrisas, sus respuestas a la voz y su enorme alegría ante las canciones que reconocen. Es posible incluso apreciar gestos de cansancio. De esta forma, acompañamos a los padres, conversamos y respondemos a sus preguntas.

Valoramos enormemente la posibilidad de interactuar con los padres y con el bebé a través de la pantalla, aspecto que se ve facilitado en la medida en que la capacidad visual del niño es suficiente para acceder a la imagen. Este formato nos posibilita mantener el contacto con las familias, entrar en sus casas e incorporar a otros miembros que, a menudo, no asisten a las sesiones. También permite la relación con las familias que viven más alejadas o que, por otras circunstancias, tienen problemas para desplazarse al CRE. Permite dar orientaciones concretas, sostener la relación y tener una continuidad frente a las dificultades. Amplía y completa, indudablemente, las posibilidades de la intervención.

Este medio nos ayuda a trabajar los tránsitos, aspecto especialmente importante cuando el niño va a salir del programa de AT y requiere cambiar el profesional que hasta ahora había sido su referente.

En cuanto a las dificultades, pensamos que el contacto es imprescindible, y más en estas primeras edades. La interacción con el bebé, al ser tan pequeño, es a través de los padres. Esta mediación permite usar la herramienta digital, pero hay aspectos que pueden pasar desapercibidos.

Nos hacemos muchas preguntas: ¿cómo relacionarnos con el niño que se aísla? ¿Qué pasará cuando no todo vaya bien?... Por otro lado, puede sobreexponerse al bebé al uso del móvil, cuestión en permanente reflexión por parte de nuestro equipo.

Pero, realmente, lo que más echamos de menos es la parte afectiva y emocional del contacto físico con el niño. 
- En cuanto a las reuniones con padres en grupo:

Las reuniones de grupos de padres se han realizado a través de la plataforma Zoom. Toma especial importancia su preparación de las reuniones, enfatizando el trabajo de diseño y dirección de estas. Tal y como se ha comentado respecto a las sesiones online, este formato permite la participación de algunas familias que, de otro modo, no hubieran podido hacerlo.

Esta herramienta, dadas sus características, hace más difícil la espontaneidad en cuanto a la interacción entre ellos dentro del grupo. Aun así, observamos reiteradamente que se establece una corriente emocional compartida que puede ser muy potente. Este es un aspecto en el que nos interesa profundizar.

- En cuanto a las actividades con familias, dirigidas a padres y niños:

Tras entrar en la etapa de confinamiento, pensamos cómo abordar nuestras actividades con el ámbito familiar en su conjunto. Planteamos una dinámica que, por haber sido valorada de forma positiva por las familias, la seguimos manteniendo hasta el día de hoy.

Destacamos las siguientes actividades: «Meriendas de AT» (una al principio y otra al final de curso), «Taller de elaboración y adaptación de cuentos» y «Actividad de Pequeartistas» de Navidad. El diseño de las mismas tiene tres fases:

- Primera fase: elaboración de la propuesta en formato digital a través de una aplicación. En ella se presenta la actividad, se hace memoria de cómo era en otros años, se propone cómo hacerla este curso y qué materiales se van a necesitar.

- Segunda fase: las familias, desde casa, llevan a cabo el proyecto, con el planteamiento y materiales que les hemos enviado. Realizan grabaciones o fotos de su experiencia para luego compartir.

- Tercera fase: se recopila el material que envían las familias y se realiza una composición que podemos compartir. 
El formato que le damos siempre es audiodescrito, con imágenes grandes y lo más simples y contrastadas posibles e instrucciones claras. Nos dirigimos a los más pequeños con un lenguaje claro y repetitivo que les ayude a poder anticipar. Usamos canciones o retahílas sencillas que puedan cantar en casa o que describan el proceso, apoyadas con gestos fáciles de realizar. Cuando ha sido necesario, le hemos añadido la LSE para alumnos con sordoceguera.

Como ejemplo, detallamos una de las actividades con más tradición, la «Merienda», que, al final de curso, llamamos «La fiesta del agua». Esta, en concreto, se lleva realizando desde hace años en nuestro centro con una gran participación. En ella colabora la especialista de música, que ha elaborado la canción guía de la actividad.

Siempre nos ha parecido un momento de convivencia entrañable. Por ello, aun en plena pandemia, pensamos en cómo adaptarla, para mantener ese espacio de diversión en el que las familias disfrutan de una actividad común. En este caso, una actividad lúdica alrededor del agua. Hubo mucha participación. Recibimos gran cantidad de vídeos y fotos mostrando cómo la habían llevado a cabo. Fue la primera actividad que adaptamos, y, dada su aceptación tanto por los padres como por los profesionales, hemos mantenido el mismo formato.

Adjuntamos el enlace al vídeo correspondiente a la primera fase: https://photos. app.goo.gl/G8cWu2VnQtSuj1LT7.

También añadimos el enlace al vídeo de una canción infantil muy conocida adaptada en LSE y en imagen con audio: https://photos.app.goo.gl/K4fRzf8nJJPkn8kp9.

\subsection{Valoración de este segundo periodo}

Recordamos otro tiempo en que pedíamos a la oftalmóloga que no recibiera a los bebés con batas médicas... Y ahora, no notamos extrañeza en ellos cuando nos ven pertrechadas con el equipo de protección individual (EPI). Vienen contentos, quizás el contexto les ayuda a interpretar. Siguen riendo con nosotros y prestan atención a nuestras canciones y palabras, entre mascarillas y gafas de protección e incluso a través de la pantalla. Vemos pocas respuestas de rechazo. Nos sonríen cuando les hablamos o saludamos y nos diferencian a unas de otras. iNos sorprenden! 
Estamos toda la mañana con el equipo de protección y, al trabajar, nos olvidamos de que lo tenemos puesto. Han cambiado muchas cosas y siguen siendo aun más importantes otras, como la coordinación y el trabajo en equipo. En definitiva, un compromiso y tarea de todos. Se hace imprescindible hasta el trabajo más invisible. Todos estamos siendo esenciales.

Las nuevas tecnologías han puesto a nuestra disposición una flexibilidad y una posibilidad de intervenir extraordinarias. Con su uso, las adaptaciones necesarias y el horizonte de la atención presencial siempre en la mente, seguimos manteniendo la mayoría de los aspectos del programa. Está claro que han venido para quedarse, y que son y serán herramientas fundamentales en nuestro trabajo.

Tras todo esto, lo más humano: «la importancia de sentirse acogido». Da igual por el medio que sea.

En cuanto a nosotras como profesionales, nos cuesta mantener las medidas de seguridad y, a la vez, seguir con los padres, los bebés y disfrutar de estar juntos, que es uno de los objetivos fundamentales de nuestro trabajo. Ponerse y quitarse el EPI, el control de la higiene y la limpieza de los materiales, el cumplimiento de los protocolos y la preocupación por las cadenas de contagio exigen un esfuerzo de atención que se suma al de la intervención propiamente dicha.

Por otro lado, destacamos que, una vez que entramos en sesión, todo se mantiene igual. La relación con los padres y el bebé prosigue la dinámica que trabajamos desde hace años. Sorprendentemente, continúa fluyendo en las sesiones el dolor ante la discapacidad de los hijos, el llanto, el juego y el asombro compartido. Además de la sonrisa, los brazos y, en definitiva, el amor.

Observamos evolución en nuestra manera de trabajar, ampliamos nuestras estructuras mentales, contemplando el trabajo online como un recurso muy potente. Complejidades compartidas, presencia y virtualidad se complementan. El trabajo nos muestra diariamente que, en estas edades, lo virtual cobra sentido solo si se asienta firmemente sobre lo concreto, sobre de la presencia real.

Finalmente, podemos decir que estamos muy satisfechas de haber conseguido mantener los aspectos básicos de la atención. Aunque, en la fecha en la que estamos, reconocemos desgaste, cansancio y preocupación. Como todos, estamos deseando 
volver a tocarnos y acercarnos con normalidad. Y, cómo no, el entusiasmo y el amor a la tarea siguen siendo igual de necesarios.

Cristina García-Trevijano Patrón. Maestra de Atención Temprana. Centro de Recursos Educativos de la ONCE en Sevilla. Campo de los Mártires, 10; 41018 Sevilla (España). Correo electrónico: cgtp@once.es.

M. ${ }^{a}$ del Carmen Hernández Mariano. Maestra de Atención Temprana. Centro de Recursos Educativos de la ONCE en Sevilla. Campo de los Mártires, 10; 41018 Sevilla (España). Correo electrónico:mchm@once.es.

Coral del Pilar Regidor Poyatos. Maestra de Atención Temprana. Centro de Recursos Educativos de la ONCE en Sevilla. Campo de los Mártires, 10; 41018 Sevilla (España). Correo electrónico: cprp@once.es.

Josefa Gómez Mateos. Maestra de Atención Temprana. Centro de Recursos Educativos de la ONCE en Sevilla. Campo de los Mártires, 10; 41018 Sevilla (España). Correo electrónico: jgom@once.es.

M. ${ }^{a}$ del Mar Martín de Frutos. Maestra de Atención Temprana. Centro de Recursos Educativos de la ONCE en Sevilla. Campo de los Mártires, 10; 41018 Sevilla (España). Correo electrónico: mmmf@once.es. 


\section{Apéndice. Proyecto de nuevo ParCRE}

\section{Rincón parque infantil «EI nuevo ParCRE»}

Lugar: patio cubierto del CRE. Entre las columnas del centro.

Materiales: casita nueva, módulo del tobogán, balancín de madera, colchoneta, saltador. Estos elementos irían puestos sobre los tatamis rosas-gris (plataformas de foam gruesas) que sean necesarios para cubrir esa zona del patio.

Responsables: programa de AT.

\section{Objetivo:}

- Disponer de un lugar al aire libre donde valorar o abordar aspectos de motricidad gruesa y de la movilidad en exteriores.

- Mantener un espacio para disfrutar del movimiento.

- Trasladar la sala de psicomotricidad de entorno, ya que se ha inhabilitado tras realizar el protocolo-covid por la alerta sanitaria.

\section{Croquis:}

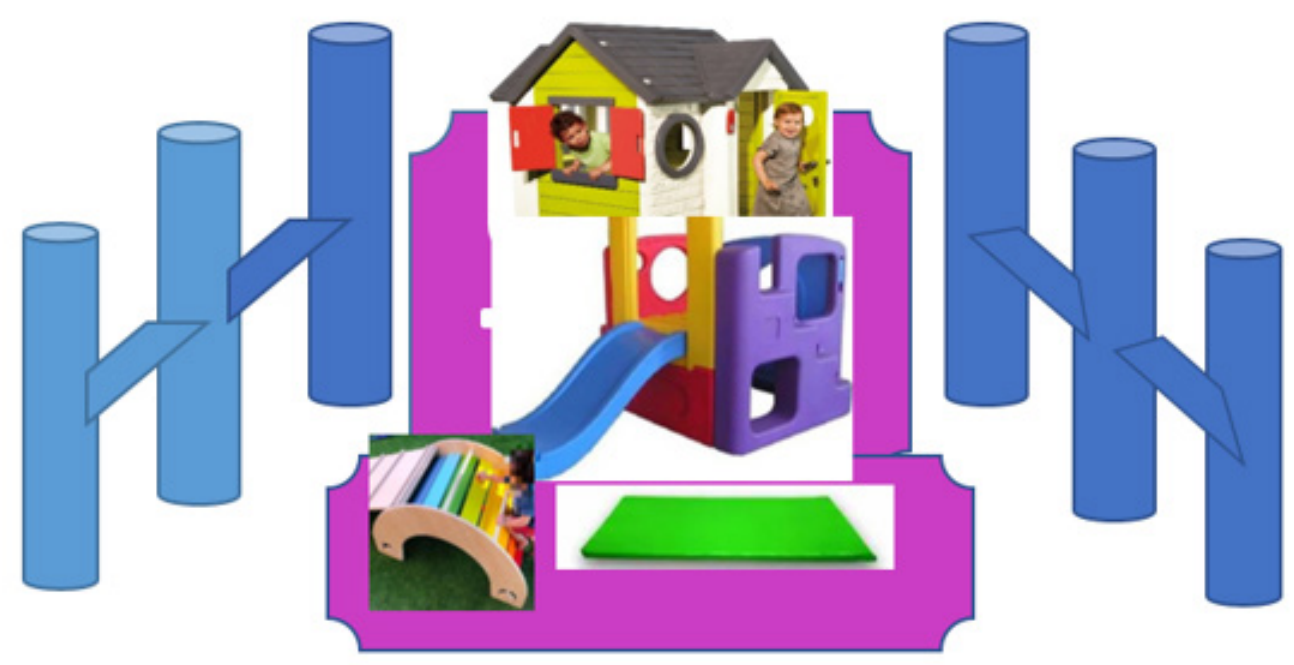

García-Trevijano, C., Hernández, M.C., Regidor, C.P., Gómez, J., Martín, M.M. (2021). Transitando por la pandemia: adaptaciones a la epidemia de la covid-19 desde el programa de Atención Temprana del CRE de Sevilla. RED Visual: Revista Especializada en Discapacidad Visual, 77, 182-199. https://doi.org/10.53094/TRIJ4448. 